\title{
Universidade de São Paulo Instituto de Química de São Carlos
}

\begin{abstract}
"Aplicação de complexos de metais de transição coordenados a típicos aditivos orgânicos de banhos eletrolíticos em eletrodeposição binária de metais"
\end{abstract}

Rogério Haruo Watanabe

Tese apresentada ao Instituto de Química de São Carlos, Universidade de São Paulo, para obtenção do título de Doutor em Ciências, na área Química Analítica.

Orientador: Prof. Dr. Benedito dos Santos Lima Neto

São Carlos

2008 
Determinação, coragem e autoconfiança são fatores decisivos para o sucesso. Não importa quais sejam os obstáculos e as dificuldades. Se estamos possuídos de uma inabalável determinação, conseguiremos superá-los. Independentemente das circunstâncias, devemos ser sempre humildes, recatados e despidos de orgulho. 
Aos meus pais, Hiroshi e Sueko e aos meus irmãos Lilian, Fábio e Cristiane, pelo incentivo e principalmente apoio durante esses anos de doutorado 


\section{Agradecimentos}

Ao Prof. Benedito pela orientação, amizade, confiança e apoio durante o tempo do meu trabalho de mestrado.

Ao Prof. Dr. Douglas Wagner Franco, Prof. Dr. Ubirajara Pereira Rodrigues e Daniel Rodrigues Cardoso pela convivência ao longo do tempo do meu mestrado.

A CAPES pelo suporte financeiro.

A minha noiva Lucimara pelo incentivo, compreensão e pelo carinho.

Aos técnicos, funcionários e vigilantes do IQSC, em especial a Veroneide e Claudia pela ajuda, auxílio e convivência.

A Mariana pela ajuda nas discussões e pela paciência em me aturar, ao Seca (Maurício) e Larissa pelas discussões.

Ao Jean, Clayston e Gustavo pela boas discussões.

Aos meus amigos de laboratório e do Grupo de Química Inorgânica Analítica: Fefê, Renata, Simone, Evânia, Gabriela, Heloísa, Daniela, Rafaela, Fernanda, Camila, Pedro, Maykon, André, Zé, Magão, Cajuru (Carlos), Itapira, Wendel, Eduardo, Negão, André, Baixinho, Fernando pelo companheirismo e pelo vários momentos de descontração.

Enfim aos meus amigos: Eurico, Morte (Adriano), Evandro, Lafon (Gay), Paulino, Josy, Maristela, Ana, Mirian, Fabiano, Tati, Jorge (Boliviano), Lisbeth, Thiago, Avelardo, Raquel, Toni, Luís, Poof (Sidnei), muito obrigado. 


\section{SUMÁRIO}

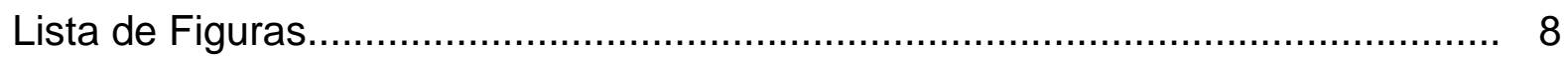

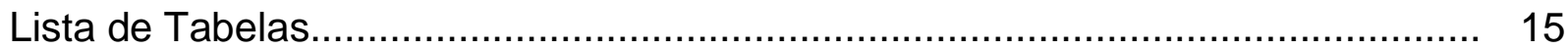

Lista de Esquemas.............................................................................. 17

Ligantes estudados............................................................................ 18

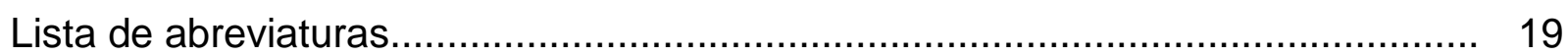

Resumo

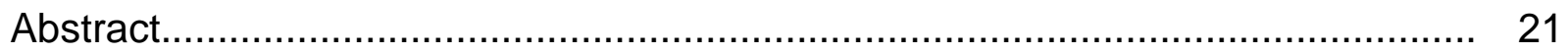

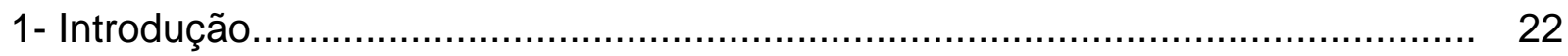

1.1- Alguns aspectos sobre os metais em estudo.............................................. 22

1.2- Química dos metais de transição e formação de complexos........................... 23

1.3- Alguns aspectos sobre eletrodeposição..................................................... 24

1.4- Estudo da importância das ligas metálicas.................................................. 25

1.5- Utilização de aditivos na indústria de eletrodeposição.................................... 27

1.6- Exemplos de estudos de eletrodeposição na presença de aditivos................ 28

1.7- Alguns aspectos sobre corrosão............................................................ 33

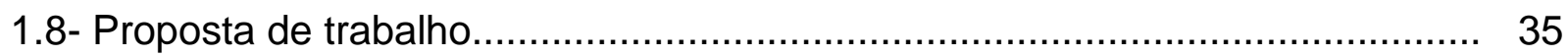

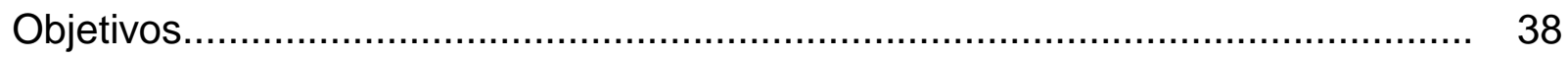

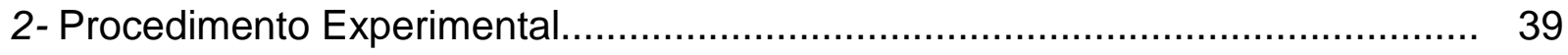

2.1- Reagentes Utilizados........................................................................ 39

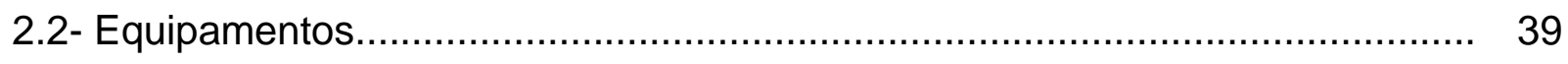

2.3- Sínteses dos Complexos...................................................................... 41

3- Resultados e Discussões...................................................................... 42 
3.1- Caracterizações dos complexos no estado sólido....................................... 42

3.1.1- Estudos dos complexos com ligante oxalato por infravermelho................. 42

3.1.2- Estudos dos complexos com o ligante etilenodiamina (EDA) por infravermelho...................................................................................... 44

3.1.3- Estudos dos complexos com o ligante citrato por infravermelho.................. 47

3.1.4- Estudos dos complexos com o ligante tetraetilenopentamina (TEPA) por infravermelho........................................................................................ 49

3.1.5- Estudos por análise elementar......................................................... 52

3.2- Caracterização dos complexos em solução................................................... 57

3.2.1 - Estudos dos por espectrofotometria de uv-visível................................... 57

3.2.2- Estudos dos complexos por voltametria cíclica........................................ 59

3.3- Estudos de eletrólise e morfologia dos depósitos......................................... 65

3.3.1- Sistemas com complexos com oxalato: $\mathrm{Cu}-\mathrm{Nb}$ e $\mathrm{Ni}-\mathrm{Nb} . \ldots \ldots \ldots \ldots \ldots \ldots \ldots \ldots \ldots \ldots . . . . . . . . . . . . . .65$

3.3.2- Sistemas com complexos de Cu-Ni com oxalato, citrato, EDA e TEPA......... 69

3.3.3- Sistemas com complexos de Cu-Zn com oxalato, citrato, EDA e TEPA........ 79

3.4- Estudos de reflectância dos depósitos....................................................... 88

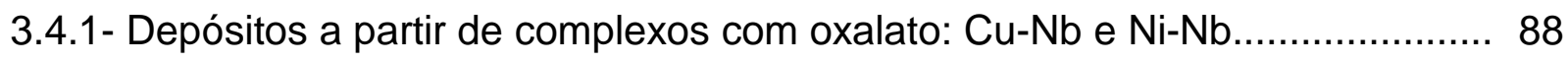

3.4.2- Depósitos a partir de complexos de $\mathrm{Cu}$ e $\mathrm{Ni}$ com os ligantes oxalato,

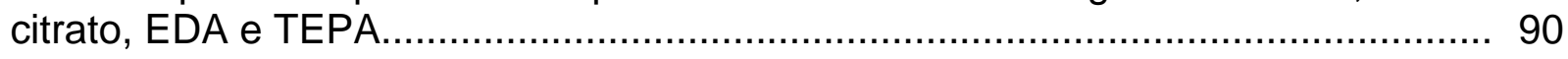

3.4.3- Depósitos a partir de complexos de Cu e Zn com os ligantes oxalato, citrato, EDA e TEPA...................................................................................... 93

3.5- Estudos de difração de raio-X dos depósitos................................................ 96

3.5.1- Depósitos de Cu-Nb e Ni-Nb obtidos a partir de complexos com oxalato...... 96

3.5.2- Depósitos de Cu-Ni obtidos a partir de complexos com oxalato, citrato, EDA e TEPA

3.5.3- Depósitos de Cu-Zn obtidos a partir de complexos com oxalato, citrato, EDA e TEPA. 
3.6- Estudos de corrosão dos depósitos........................................................ 110

3.6.1- Sistemas com complexos de $\mathrm{Cu}, \mathrm{Ni}$ e $\mathrm{Nb}$ com oxalato.............................. 110

3.6.2- Sistemas com complexos de Cu-Ni com oxalato, citrato, EDA e TEPA........ 113

3.6.3- Sistemas com complexos de Cu-Zn com oxalato, citrato, EDA e TEPA....... 115

3.7- Estudos de comparação de eficiência dos complexos gerados ex-situ com os 119

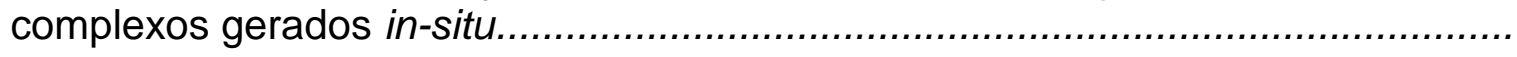

3.7.1- Depósitos do filme Cu-Nb e Ni-Nb obtidos de complexos gerados ex-situ e

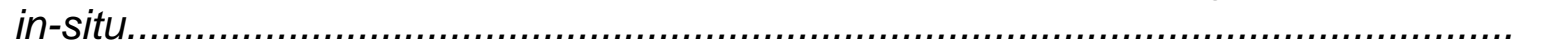

3.7.2- Depósitos do filme Cu-Ni obtidos de complexos gerados ex-situ e in-situ..... 124

3.7.3- Depósitos do filme Cu-Zn obtidos de complexos gerados ex-situ e in-situ.... 128

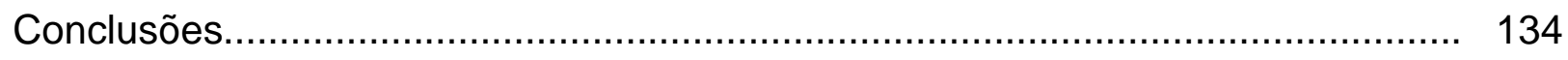

Referências bibliográficas...................................................................... 137 


\section{Lista de Figuras}

Figura 1: Espectros de infravermelho do complexo Cu-oxalato e dos sais de partida, em pastilhas de $\mathrm{KBr}$.

Figura 2: Espectros de infravermelho do complexo Ni-oxalato e dos sais de partida, em pastilhas de $\mathrm{KBr}$.

Figura 3: Espectros de infravermelho do complexo $\mathrm{Zn}$-oxalato e dos sais de partida, em pastilhas de $\mathrm{KBr}$

Figura 4: Espectros de infravermelho do complexo Cu-EDA e dos sais de partida, em pastilhas de $\mathrm{KBr}$

Figura 5: Espectros de infravermelho do complexo Ni-EDA e dos sais de partida, em pastilhas de $\mathrm{KBr}$.

Figura 6: Espectros de infravermelho do complexo Zn-EDA e dos sais de partida, em pastilhas de $\mathrm{KBr}$

Figura 7: Espectros de infravermelho do complexo Cu-citrato e dos sais de partida, em pastilhas de $\mathrm{KBr}$.

Figura 8: Espectros de infravermelho do complexo Ni-citrato e dos sais de partida, em pastilhas de $\mathrm{KBr}$.

Figura 9: Espectros de infravermelho do complexo Zn-citrato e dos sais de partida, em pastilhas de $\mathrm{KBr}$

Figura 10: Espectros de infravermelho do complexo Cu-TEPA e dos sais de partida, em pastilhas de $\mathrm{KBr}$.

Figura 11: Espectros de infravermelho do complexo Ni-TEPA e dos sais de partida, em pastilhas de $\mathrm{KBr}$

Figura 12: Espectros de infravermelho do complexo Zn-TEPA e dos sais de partida, em pastilhas de $\mathrm{KBr}$

Figura 13: Espectro eletrônico dos complexos Cu-oxalato, Cu-EDA, Cu-citrato, Cu-TEPA e do $\mathrm{CuSO}_{4}$ em água.

Figura 14: Espectro-eletrônico dos complexos Ni-oxalato, Ni-EDA, Ni-citrato, $\mathrm{Ni}$ TEPA e do $\mathrm{NiSO}_{4}$ em água 
Figura 15: Voltametria cíclica das soluções do $\mathrm{CuSO}_{4}$ e dos complexos $\mathrm{Cu}$ oxalato e Cu-citrato em substrato de platina; $\mu=0,5\left(\mathrm{Na}_{2} \mathrm{SO}_{4}\right), \mathrm{pH}=4,0\left(\mathrm{H}_{2} \mathrm{SO}_{4}\right)$ $\mathrm{e} v=50 \mathrm{mVs}^{-1}$

Figura 16: Voltametria cíclica das soluções do $\mathrm{CuSO}_{4}$ e dos complexos $\mathrm{Cu}$-EDA e Cu-TEPA em substrato de platina; $\mu=0,5\left(\mathrm{Na}_{2} \mathrm{SO}_{4}\right), \mathrm{pH}=4,0\left(\mathrm{H}_{2} \mathrm{SO}_{4}\right)$ e $\mathrm{v}=50$ $\mathrm{mVs}^{-1}$

Figura 17: Voltametria cíclica das soluções do $\mathrm{NiSO}_{4} \mathrm{e}$ dos complexos $\mathrm{Ni}$-oxalato e Ni-citrato em substrato de platina; $\mu=0,5\left(\mathrm{Na}_{2} \mathrm{SO}_{4}\right), \mathrm{pH}=4,0\left(\mathrm{H}_{2} \mathrm{SO}_{4}\right)$ e $v=50$ $\mathrm{mVs}^{-1}$

Figura 18: Voltametria cíclica das soluções do $\mathrm{NiSO}_{4}$ e dos complexos $\mathrm{Ni}$-EDA e Ni-TEPA em substrato de platina; $\mu=0,5\left(\mathrm{Na}_{2} \mathrm{SO}_{4}\right), \mathrm{pH}=4,0\left(\mathrm{H}_{2} \mathrm{SO}_{4}\right)$ e $v=50$ $\mathrm{mVs}^{-1}$

Figura 19: Voltametria cíclica das soluções do $\mathrm{ZnSO}_{4}$ e dos complexos $\mathrm{Zn}$ oxalato e $\mathrm{Zn}$-citrato em substrato de platina; $\mu=0,5\left(\mathrm{Na}_{2} \mathrm{SO}_{4}\right), \mathrm{pH}=4,0\left(\mathrm{H}_{2} \mathrm{SO}_{4}\right)$ $\mathrm{e} v=50 \mathrm{mVs}^{-1}$

Figura 20: Voltametria cíclica das soluções do $\mathrm{ZnSO}_{4}$ e dos complexos $\mathrm{Zn}$-EDA e $\mathrm{Zn}$-TEPA em substrato de platina; $\mu=0,5\left(\mathrm{Na}_{2} \mathrm{SO}_{4}\right), \mathrm{pH}=4,0\left(\mathrm{H}_{2} \mathrm{SO}_{4}\right)$ e $v=50$ $\mathrm{mVs}^{-1}$

Figura 21: Voltamogramas das soluções do Cu-oxalato, Nb-oxalato e $\mathrm{Cu}-\mathrm{Nb}$ oxalato em eletrodo de aço 1010, (Cu(II) 5,6 mmol..-1, $\mathrm{Nb}(\mathrm{V}) 17 \mathrm{mmol}^{-\mathrm{L}^{-1}}$ ); $\mu=$ $0,5\left(\mathrm{Na}_{2} \mathrm{SO}_{4}\right), \mathrm{pH}=4,0\left(\mathrm{H}_{2} \mathrm{SO}_{4}\right), \mathrm{v}=50 \mathrm{mVs}^{-1}$

Figura 22: Voltamogramas das soluções do $\mathrm{Ni}$-oxalato, $\mathrm{Nb}$-oxalato e $\mathrm{Ni}-\mathrm{Nb}$ oxalato em eletrodo de aço 1010, (Ni(II) 9,9 mmol..-1 $, \mathrm{Nb}(\mathrm{V}) 3,3 \mathrm{mmol} . \mathrm{L}^{-1}$ ); $\mu=$ $0,5\left(\mathrm{Na}_{2} \mathrm{SO}_{4}\right), \mathrm{pH}=4,0\left(\mathrm{H}_{2} \mathrm{SO}_{4}\right), \mathrm{v}=50 \mathrm{mVs}^{-1}$

Figura 23: Micrografias dos depósitos gerados a partir da solução $\mathrm{Cu}$-Nb-oxalato em eletrodo de aço 1010 (Cu(II) 5,6 mmol.L $\mathrm{L}^{-1}, \mathrm{Nb}(\mathrm{V}) 17 \mathrm{mmol} . \mathrm{L}^{-1}$ )

Figura 24: Micrografias dos depósitos gerados a partir da solução $\mathrm{Ni}-\mathrm{Nb}$-oxalato em eletrodo de aço 1010. (Ni (II) 9,9 mmol. $\mathrm{L}^{-1}, \mathrm{Nb} 3,3 \mathrm{mmol}^{-1} \mathrm{~L}^{-1}$ )

Figura 25: Voltamograma das soluções do Cu-oxalato, Ni-oxalato e $\mathrm{Cu}-\mathrm{Ni}$ oxalato em eletrodo de aço 1010, (Cu(II) 5,7 mmol. $\mathrm{L}^{-1}$, Ni(II) $16,8 \mathrm{mmol} . \mathrm{L}^{-1}$ ); $\mu=$ $0,5\left(\mathrm{Na}_{2} \mathrm{SO}_{4}\right), \mathrm{pH}=4,0\left(\mathrm{H}_{2} \mathrm{SO}_{4}\right), \mathrm{v}=50 \mathrm{mVs}^{-1} \ldots$

Figura 26: Voltamograma das soluções do Cu-citrato, Ni-citrato e Cu-Ni-citrato em eletrodo de aço 1010, (Cu(II) 6,1 mmol. $\mathrm{L}^{-1}$, Ni(II) $18,5 \mathrm{mmol} . \mathrm{L}^{-1}$ ); $\mu=0,5$ $\left(\mathrm{Na}_{2} \mathrm{SO}_{4}\right), \mathrm{pH}=4,0\left(\mathrm{H}_{2} \mathrm{SO}_{4}\right), \mathrm{v}=50 \mathrm{mVs}^{-1}$ 
Figura 27: Voltamograma das soluções do Cu-EDA, Ni-EDA e Cu-Ni-EDA em eletrodo de aço 1010, (Cu(II) 12,6 mmol..-1 $\mathrm{Ni}(\mathrm{II})$ 12,5 mmol. $\mathrm{L}^{-1}$ ); $\mu=0,5$ $\left(\mathrm{Na}_{2} \mathrm{SO}_{4}\right), \mathrm{pH}=4,0\left(\mathrm{H}_{2} \mathrm{SO}_{4}\right), \mathrm{v}=50 \mathrm{mVs}^{-1}$

Figura 28: Voltamograma das soluções do Cu-TEPA, Ni-TEPA e Cu-Ni-TEPA em eletrodo de aço 1010, (Cu(II) 9,9 mmol.L ${ }^{-1}$, Ni(II) 19,8 mmol..-1 $\left.{ }^{-1}\right) ;=0,5$ $\left(\mathrm{Na}_{2} \mathrm{SO}_{4}\right), \mathrm{pH}=4,0\left(\mathrm{H}_{2} \mathrm{SO}_{4}\right), \mathrm{v}=50 \mathrm{mVs}^{-1}$

Figura 29: Micrografias dos depósitos gerados a partir da solução Cu-Ni-oxalato em eletrodo de aço 1010. (Cu(II) 5,7 mmol. $\mathrm{L}^{-1}$, Ni(II) 16,8 mmol.. ${ }^{-1}$ ).

Figura 30: Micrografias dos depósitos gerados a partir da solução $\mathrm{Cu}-\mathrm{Ni}$-citrato em eletrodo de aço 1010 (Cu(II) 6,1 mmol..-1 ${ }^{-1} \mathrm{Ni}(\mathrm{II})$ 18,5 mmol..-1 $)$

Figura 31: Micrografias dos depósitos gerados a partir da solução Cu-Ni-EDA em eletrodo de aço 1010 (Cu(II) 12,6 mmol.L $\mathrm{L}^{-1}$, Ni(II) 12,5 mmol..-1 $)$.

Figura 32: Micrografias dos depósitos gerados a partir da solução Cu-Ni-TEPA em eletrodo de aço $1010[\mathrm{Cu}(\mathrm{II})]=9,9 \mathrm{mmol} \cdot \mathrm{L}^{-1}$, [Ni(II)] = 19,8 mmol. $\mathrm{L}^{-1}$.

Figura 33: Voltamograma das soluções do Cu-oxalato, Zn-oxalato e Cu-Znoxalato em eletrodo de aço 1010, (Cu(II) 5,7mmol.L $\mathrm{L}^{-1}, \mathrm{Zn}$ (II) $16,9 \mathrm{mmol}^{-1} \mathrm{~L}^{-1}$ ); $\mu=$ $0,5\left(\mathrm{Na}_{2} \mathrm{SO}_{4}\right), \mathrm{pH}=4,0\left(\mathrm{H}_{2} \mathrm{SO}_{4}\right), \mathrm{v}=50 \mathrm{mVs}^{-1}$

Figura 34: Voltamograma das soluções do Cu-citrato, Zn-citrato e Cu-Zn-citrato em eletrodo de aço 1010, (Cu(II) 6,1 mmol..-1 $\mathrm{Zn}$ (II) 18,5 mmol..-1 ) $^{-1} \mu=0,5$ $\left(\mathrm{Na}_{2} \mathrm{SO}_{4}\right), \mathrm{pH}=4,0\left(\mathrm{H}_{2} \mathrm{SO}_{4}\right), \mathrm{v}=50 \mathrm{mVs}^{-1}$

Figura 35: Voltamograma das soluções do Cu-EDA, Zn-EDA e Cu-Zn-EDA em eletrodo de aço 1010, (Cu(II) 12,6 mmol. $\left.\mathrm{L}^{-1}, \mathrm{Zn}(\mathrm{II}) 12,6 \mathrm{mmol} . \mathrm{L}^{-1}\right) ; \mu=0,5$ $\left(\mathrm{Na}_{2} \mathrm{SO}_{4}\right), \mathrm{pH}=4,0\left(\mathrm{H}_{2} \mathrm{SO}_{4}\right), \mathrm{v}=50 \mathrm{mVs}^{-1}$

Figura 36: Voltamograma das soluções do Cu-TEPA, Zn-TEPA e Cu-Zn-TEPA em eletrodo de aço 1010, (Cu(II) 9,9 mmol.L-1 $\mathrm{Zn}$ (II) 19,8 mmol.. ${ }^{-1}$ ); $\mu=0,5$ $\left(\mathrm{Na}_{2} \mathrm{SO}_{4}\right), \mathrm{pH}=4,0\left(\mathrm{H}_{2} \mathrm{SO}_{4}\right), \mathrm{v}=50 \mathrm{mVs}^{-1}$.

Figura 37: Micrografias dos depósitos gerados a partir da solução $\mathrm{Cu}$-Zn-oxalato em eletrodo de aço 1010. (Cu(II) 5,7 mmol..-1, $\mathrm{Zn}$ (II) 16,9 mmol. $\mathrm{L}^{-1}$ ).

Figura 38: Micrografias dos depósitos gerados a partir da solução Cu-Zn-citrato em eletrodo de aço 1010. (Cu(II) 6,1 mmol. $\mathrm{L}^{-1}, \mathrm{Zn}$ (II) 18,5 mmol.. ${ }^{-1}$ )

Figura 39: Micrografias dos depósitos gerados a partir da solução Cu-Zn-EDA em eletrodo de aço 1010. (Cu(II) 12,6 mmol..-1, Zn(II) 12,6 mmol.. ${ }^{-1}$ ) 
Figura 40: Micrografias dos depósitos gerados a partir da solução Cu-Zn-TEPA em eletrodo de aço 1010. (Cu(II) 9,9 mmol.. ${ }^{-1}, \mathrm{Zn}$ (II) 19,8 mmol.. ${ }^{-1}$ )....................

Figura 41: Curvas de reflectância dos depósitos obtidos dos banhos Cu-oxalato, $\mathrm{Nb}$-oxalato e Cu-Nb-oxalato ( $E_{\text {aplic. }}=-1,29 \mathrm{~V}$ )em eletrodo de aço 1010..................

Figura 42: Curvas de reflectância dos depósitos obtidos dos banhos Ni-oxalato, $\mathrm{Nb}$-oxalato e $\mathrm{Ni}-\mathrm{Nb}$-oxalato $\left(E_{\text {aplic. }}=-1,15 \mathrm{~V}\right.$ ) em eletrodo de aço 1010.

Figura 43: Curvas de reflectância dos depósitos obtidos dos banhos Cu-oxalato,

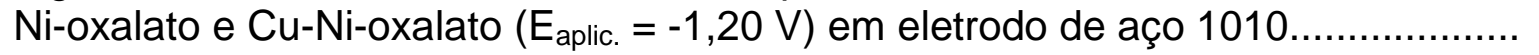

Figura 44: Curvas de reflectância dos depósitos obtidos dos banhos Cu-citrato, Ni-citrato e Cu-Ni-citrato ( $E_{\text {aplic. }}=-1,40 \mathrm{~V}$ ) em eletrodo de aço 1010......................

Figura 45: Curvas de reflectância dos depósitos obtidos dos banhos Cu-EDA, NiEDA e Cu-Ni-EDA ( $E_{\text {aplic. }}=-1,60 \mathrm{~V}$ ) em eletrodo de aço 1010.

Figura 46: Curvas de reflectância dos depósitos obtidos dos banhos Cu-TEPA, $\mathrm{Ni}$-TEPA e Cu-Ni-TEPA ( $E_{\text {aplic. }}=-1,50 \mathrm{~V}$ ) em eletrodo de aço 1010.

Figura 47: Curvas de reflectância dos depósitos obtidos dos banhos Cu-oxalato, Zn-oxalato e Cu-Zn-oxalato ( $E_{\text {aplic. }}=-1,50 \mathrm{~V}$ ) em eletrodo de aço 1010.

Figura 48: Curvas de reflectância dos depósitos dos banhos Cu-citrato, Zn-citrato e Cu-Zn-citrato $\left(E_{\text {aplic. }}=-1,50 \mathrm{~V}\right)$ em eletrodo de aço 1010.

Figura 49: Curvas de reflectância dos depósitos obtidos dos banhos Cu-EDA, ZnEDA e Cu-Zn-EDA ( $E_{\text {aplic. }}=-1,50 \mathrm{~V}$ ) em eletrodo de aço 1010..............................

Figura 50: Curvas de reflectância dos depósitos obtidos dos banhos Cu-TEPA, Zn-TEPA e Cu-Zn-TEPA ( $E_{\text {aplic. }}=-1,60$ V) em eletrodo de aço 1010.

Figura 51: Difratograma de raio-X do depósito obtido do sistema Cu-Nb-oxalato em $-1,29 \mathrm{~V}$ com $300 \mathrm{mC}$ em eletrodo de aço.

Figura 52: Difratograma de raio- $\mathrm{X}$ do depósito obtido do sistema $\mathrm{Ni-Nb-oxalato}$ em $-1,15 \mathrm{~V}$ com $300 \mathrm{mC}$ em eletrodo de aço.....................................................

Figura 53: Difratograma de raio-X do depósito obtido do sistema Cu-Ni-oxalato em $-1,20 \mathrm{~V}$ com $300 \mathrm{mC}$ em eletrodo de aço. 100

Figura 54: Difratograma de raio-X do depósito obtido do sistema Cu-Ni-citrato em $-1,40 \mathrm{~V}$ com $300 \mathrm{mC}$ em eletrodo de aço. 
Figura 55: Difratograma de raio-X do depósito obtido do sistema Cu-Ni-EDA em $1,60 \mathrm{~V}$ com $300 \mathrm{mC}$ em eletrodo de aço.

Figura 56: Difratograma de raio-X do depósito obtido do sistema Cu-Ni-TEPA em $-1,50 \mathrm{~V}$ com $300 \mathrm{mC}$ em eletrodo de aço.

Figura 57: Difratograma de raio-X do depósito obtido do sistema $\mathrm{Cu}-\mathrm{Zn}$-oxalato em $-1,50 \mathrm{~V}$ com $300 \mathrm{mC}$ em eletrodo de aço.

Figura 58: Difratograma de raio-X do depósito obtido do sistema $\mathrm{Cu}-\mathrm{Zn}$-citrato em $-1,50 \mathrm{~V}$ com $300 \mathrm{mC}$ em eletrodo de aço.

Figura 59: Difratograma de raio-X do depósito obtido do sistema Cu-Zn-EDA em $-1,50 \mathrm{~V}$ com $300 \mathrm{mC}$ em eletrodo de aço

Figura 60: Difratograma de raio-X do depósito obtido do sistema Cu-Zn-TEPA em $-1,60 \mathrm{~V}$ com $300 \mathrm{mC}$ em eletrodo de aço

Figura 61: Curvas de polarização anódica dos substratos de aço $1010 \mathrm{sem}$ e com os filmes contendo os elementos $\mathrm{Cu} / \mathrm{Nb}$ ou $\mathrm{Ni} / \mathrm{Nb}$.

Figura 62: Curva de polarização dos substratos de aço 1010 sem e com os filmes contendo os elementos $\mathrm{Cu} / \mathrm{Nb}$ ou $\mathrm{Ni} / \mathrm{Nb}$.

Figura 63: Curvas de polarização anódica dos substratos de aço 1010 sem e com os filmes contendo os elementos $\mathrm{Cu} / \mathrm{Ni}$.

Figura 64: Curva de polarização dos substratos de aço 1010 sem e com os filmes contendo os elementos $\mathrm{Cu} / \mathrm{Ni}$.

Figura 65: Curvas de polarização anódica dos substratos de aço $1010 \mathrm{sem}$ e com os filmes contendo os elementos $\mathrm{Cu} / \mathrm{Zn}$.

Figura 66: Curva de polarização dos substratos de aço 1010 sem e com os filmes contendo os elementos $\mathrm{Cu} / \mathrm{Zn}$

Figura 67: Micrografias dos depósitos gerados a partir dos complexos gerados ex-situ (A) e in-situ (B) da solução Cu-Nb-oxalato em eletrodo de aço 1010

Figura 68: Micrografias dos depósitos gerados a partir dos complexos gerados ex-situ (A) e in-situ (B) da solução $\mathrm{Ni}$-Nb-oxalato em eletrodo de aço 1010

Figura 69: Curvas de polarização anódica dos substratos de aço 1010 sem e com os filmes contendo os elementos $\mathrm{Cu} / \mathrm{Nb}$ ou $\mathrm{Ni} / \mathrm{Nb}$ proveniente de complexos gerados ex-situ e in-situ. 
Figura 70: Curva de polarização dos substratos de aço 1010 sem e com os filmes contendo os elementos $\mathrm{Cu} / \mathrm{Nb}$ ou $\mathrm{Ni} / \mathrm{Nb}$ proveniente de complexos gerados ex-situ e in-situ.

Figura 71: Micrografias dos depósitos gerados a partir dos complexos gerados ex-situ (A) e in-situ (B) da solução Cu-Ni-oxalato em eletrodo de aço 1010

Figura 72: Micrografias dos depósitos gerados a partir dos complexos gerados ex-situ (A) e in-situ (B) da solução Cu-Ni-citrato em eletrodo de aço 1010

Figura 73: Micrografias dos depósitos gerados a partir dos complexos gerados ex-situ (A) e in-situ (B) da solução Cu-Ni-EDA em eletrodo de aço 1010.

Figura 74: Micrografias dos depósitos gerados a partir dos complexos gerados ex-situ (A) e in-situ (B) da solução Cu-Ni-citrato em eletrodo de aço 1010.............

Figura 75: Curvas de polarização anódica dos substratos de aço $1010 \mathrm{sem} \mathrm{e}$ com os filmes contendo os elementos $\mathrm{Cu} / \mathrm{Ni}$ proveniente de complexos gerados ex-situ e in-situ.

Figura 76: Curva de polarização dos substratos de aço 1010 sem e com os filmes contendo os elementos $\mathrm{Cu} / \mathrm{Ni}$ proveniente de complexos gerados ex-situ e in-situ.

Figura 77: Micrografias dos depósitos gerados a partir dos complexos gerados ex-situ (A) e in-situ (B) da solução Cu-Zn-oxalato em eletrodo de aço 1010

Figura 78: Micrografias dos depósitos gerados a partir dos complexos gerados ex-situ (A) e in-situ (B) da solução Cu-Zn-citrato em eletrodo de aço 1010.............

Figura 79: Micrografias dos depósitos gerados a partir dos complexos gerados ex-situ (A) e in-situ (B) da solução Cu-Zn-EDA em eletrodo de aço 1010.

Figura 80: Micrografias dos depósitos gerados a partir dos complexos gerados ex-situ (A) e in-situ (B) da solução Cu-Zn-TEPA em eletrodo de aço 1010

Figura 81: Curvas de polarização anódica dos substratos de aço 1010 sem e com os filmes contendo os elementos $\mathrm{Cu} / \mathrm{Zn}$ proveniente de complexos gerados ex-situ e in-situ.

Figura 82: Curva de polarização dos substratos de aço 1010 sem e com os filmes contendo os elementos $\mathrm{Cu} / \mathrm{Zn}$ proveniente de complexos gerados ex-situ e in-situ. 


\section{Lista de Tabelas}

Tabela 1: Resultados da análise elementar $(\mathrm{C}, \mathrm{H}, \mathrm{N})$ dos complexos obtidos......

ГTabela 2: Valores de $\mathrm{E}_{\text {red. }}$ de cada complexo e dos sistemas $\mathrm{Cu}-\mathrm{Nb}$ e $\mathrm{Ni}-\mathrm{Nb}$ em aço 1010 .

Tabela 3: Resultados de EDX e aspectos morfológico para filmes $\mathrm{Cu}-\mathrm{Nb}$ e $\mathrm{Ni}$ $\mathrm{Nb}$ sobre aço 1010 em função do $\mathrm{E}_{\text {aplic., }}$, a partir de complexos com oxalato

Tabela 4: Valores de $E_{\text {red. }}$ dos complexos e dos sistemas Cu-Ni em aço 1010.....

70

Tabela 5: Resultados de EDX e aspectos morfológicos para filmes Cu-Ni sobre aço 1010 em função do $E_{\text {aplic., }}$ a partir de complexos com oxalato, citrato, EDA e TEPA.

Tabela 6: Valores de $E_{\text {red. }}$ dos complexos e dos sistemas Cu-Zn em aço 1010....

Tabela 7: Resultados de EDX e aspectos morfológicos para filmes Cu-Zn sobre aço 1010 em função do $E_{\text {aplic., }}$ a partir de complexos com oxalato, citrato, EDA e TEPA.

Tabela 8: Valores de $d(h \mathrm{kl})$ referente ao depósito proveniente do sistema Cu$\mathrm{Nb}$-oxalato.

Tabela 9: Valores de $d(h \mathrm{kl})$ referente ao depósito proveniente do sistema $\mathrm{Ni}$ $\mathrm{Nb}$-oxalato

Tabela 10: Valores de $d(h k l)$ referente ao depósito de Cu-Ni proveniente do sistema Cu-Ni-oxalato.

Tabela 11: Valores de $d(h k l)$ referente ao depósito de Cu-Ni proveniente do sistema Cu-Ni-citrato.

Tabela 12: Valores de $d(h k$ l) referente ao depósito de Cu-Ni proveniente do sistema Cu-Ni-EDA.

Tabela 13: Valores de $d(h k l)$ referente ao depósito de Cu-Ni proveniente do sistema Cu-Ni-TEPA.

Tabela 14: Valores de $d(h k l)$ referente ao depósito de Cu-Zn proveniente do sistema Cu-Zn-oxalato.

Tabela 15: Valores de $d(h \mathrm{k}$ l) referente ao depósito de Cu-Zn proveniente do sistema Cu-Zn-citrato. 
Tabela 16: Valores de $d(h \mathrm{k} l)$ referente ao depósito de Cu-Zn proveniente do sistema Cu-Zn-EDA.

Tabela 17: Valores de $d(h k l)$ referente ao depósito de Cu-Zn proveniente do sistema Cu-Zn-TEPA.

Tabela 18: Valores de $E_{\text {corr }}, E_{\text {pite }}$ e j $j_{\text {corr. }}$ do substrato de aço 1010 com e sem os filmes contendo os elementos $\mathrm{Cu} / \mathrm{Nb}$ e Ni/Nb.

Tabela 19: Valores de $E_{\text {corr }}, E_{\text {pite }}$ e j jorr. do substrato de aço 1010 com e sem os filmes contendo os elementos $\mathrm{Cu} / \mathrm{Ni}$.

Tabela 20: Valores de $E_{\text {corr }}, E_{\text {pite }}$ e $j_{\text {corr. }}$ do substrato de aço 1010 com e sem os filmes contendo os elementos $\mathrm{Cu} / \mathrm{Zn}$.

Tabela 21: Valores de $E_{\text {corr, }} E_{\text {pite }} j_{\text {corr. }}$ do substrato de aço 1010 com e sem os filmes contendo os elementos $\mathrm{Cu} / \mathrm{Nb}$ e $\mathrm{Ni} / \mathrm{Nb}$ obtidos de complexos ex-situ e insitu.

Tabela 22: Valores de $E_{\text {corr }}, E_{\text {pite }}$ e j $j_{\text {corr. }}$ do substrato de aço 1010 com e sem os filmes contendo os elementos $\mathrm{Cu} / \mathrm{Ni}$ obtidos de complexos ex-situ e insitu.

Tabela 23: Valores de $E_{\text {corr }}, E_{\text {pite }}$ e jcorr. do substrato de aço 1010 com e sem os filmes contendo os elementos $\mathrm{Cu} / \mathrm{Zn}$ obtidos de complexos ex-situ e insitu. 


\section{Lista de esquemas}

Esquema 1: Estrutura molecular da uréia e da tiouréia................................... 28

Esquema 2: Proposta para o complexo Cu-oxalato........................................ 53

Esquema 3: Proposta para o complexo Ni-oxalato......................................... 53

Esquema 4: Proposta para o complexo Zn-oxalato....................................... 54

Esquema 5: Proposta para o complexo Cu-EDA.......................................... 54

Esquema 6: Proposta para o complexo Ni-EDA........................................... 54

Esquema 7: Proposta para o complexo Zn-EDA......................................... 55

Esquema 8: Proposta para o complexo Cu-TEPA......................................... 55

Esquema 9: Proposta para o complexo Ni-TEPA......................................... 55

Esquema 10: Proposta para o complexo Zn-TEPA......................................... 56

Esquema 11: Proposta para o complexo Cu-citrato........................................ 56

Esquema 12: Proposta para o complexo Ni-citrato....................................... 56

Esquema 13: Proposta para o complexo Zn-citrato........................................ 57 


\section{Ligantes estudados}

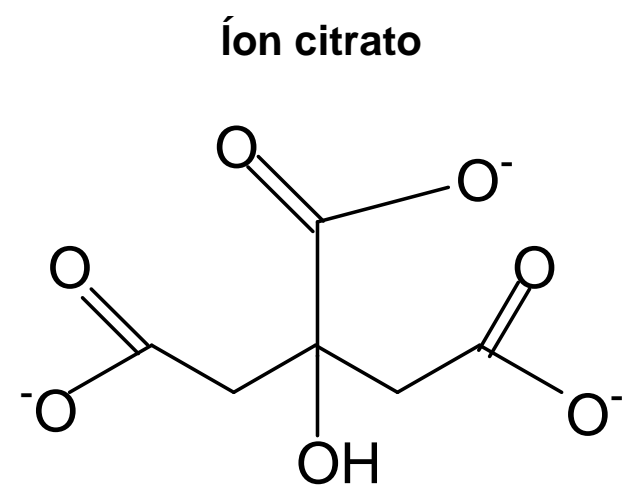

Íon oxalato

Tetraetilenopentamina (TEPA)

Etilenodiamina (EDA)
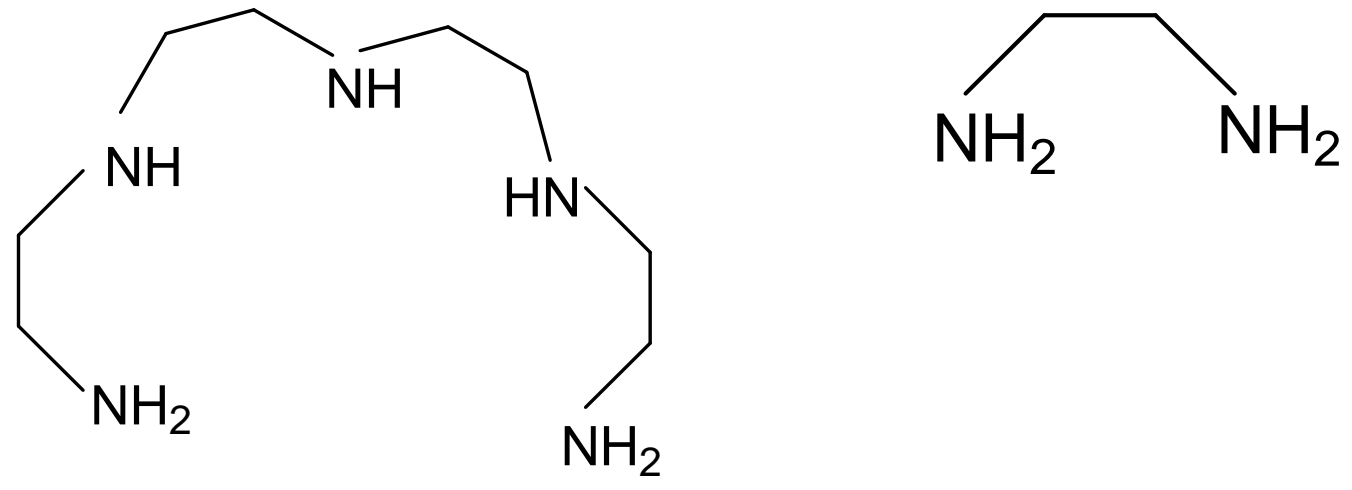


\section{Lista de Abreviaturas}

$\begin{array}{cc}\text { Palavras } & \text { Abreviação } \\ \text { Etilenodiamina } & \text { EDA } \\ \text { Tetraetilenopentamina } & \text { TEPA } \\ \text { Microscopia Eletrônica de Varredura } & \text { MEV } \\ \text { Espectroscopia de Dispersão de raio-X } & \text { EDX }\end{array}$




\section{Resumo}

O objetivo principal deste trabalho é aplicar complexos de cobre(II), níquel(II), zinco(II) e nióbio(V) como fontes de metais em banhos de eletrodeposição.

Os íons metálicos foram coordenados aos ligantes íon oxalato, íon citrato, etilenodiamina (EDA) ou tetraetilenopentamina (TEPA), os quais são aditivos orgânicos típicos em banhos de eletrodeposição.

Os complexos foram caracterizados por análise elementar, espectroscopias nas regiões do infravermelho e ultravioleta-visível e voltametria cíclica.

Foram realizadas eletrólises em presença de dois complexos de coordenação em ausência de quantias adicionais de aditivos, usando aço 1010 como substrato a pH $=4.5\left(\mathrm{H}_{2} \mathrm{SO}_{4} / \mathrm{Na}_{2} \mathrm{SO}_{4}\right)$, resultando em depósitos com dois elementos metálicos.

Os depósitos apresentaram aspectos morfológicos com boas qualidades e sem falhas. Em adição, os depósitos foram analisados por EDX, reflectância difusa e espectroscopia de raio-X e também foram realizadas medidas de curvas de polarização.

Os depósitos obtidos a partir dos complexos gerados ex-situ mostraram morfologias melhores do que depósitos obtidos de soluções preparadas com os sais dos metais na presença dos aditivos, mediante as mesmas condições de trabalho.

É sugerido que os metais de partida, coordenados a aditivos influenciam o processo de eletrodeposição, propiciando depósitos com potenciais de corrosão deslocados por até $+200 \mathrm{mV}$ em $0.5 \mathrm{~mol} \cdot \mathrm{L}^{-1} \mathrm{NaCl}\left(1 \mathrm{mV} \cdot \mathrm{s}^{-1}\right)$. 


\section{Abstract}

The main goal of this work is to use copper(II), nickel(II), zinc(II) and niobium(V) complexes as metal plating source in electrodeposition baths.

The metal ions were coordinated with oxalate, citrate, ethylenediamine (EDA) or tetraethylenepentamine (TEPA) as ligands, which are typical organic additives in electroplating. The complexes were characterized by elemental analysis, infrared and ultraviolet spectroscopies and cyclic voltammetry.

The electrolyses in presence of two coordination complexes in the baths were carried out in absence of extra amount of additive, using 1010 steel as substrate at $\mathrm{pH}=$ $4.5\left(\mathrm{H}_{2} \mathrm{SO}_{4} / \mathrm{Na}_{2} \mathrm{SO}_{4}\right)$.

Deposits with two metal elements from the ex-situ generated complexes showed good quality morphologic aspect without crashes. Further, the deposits were analyzed by EDX, diffuse reflectance and X-ray spectroscopy and polarization curves were recorded.

The deposits showed better morphologies than deposits obtained from the metal salt solutions in presence of additives under similar conditions.

It is suggestive that the starting metal plating coordinated with additives influences the electrodeposition processes, affording deposits with corrosion potentials shifted over $+200 \mathrm{mV}$ in $0.5 \mathrm{~mol} . \mathrm{L}^{-1} \mathrm{NaCl}\left(1 \mathrm{mV} . \mathrm{s}^{-1}\right)$. 